\title{
Robust Multiobjective Model Predictive Control with Computation Delay Compensation for Electric Vehicle Applications Using PMSM with Multilevel Inverter
}

\author{
Abdiddaim Katkout $\mathbb{D D}^{1}{ }^{1}$ Tamou Nasser, ${ }^{2}$ and Ahmed Essadki ${ }^{1}$ \\ ${ }^{1}$ ERERA, Research Center in Sciences and Technologies of Engineering and Health (STIS), ENSET, Mohammed V University, \\ Rabat, Morocco \\ ${ }^{2}$ ERERA, Research Center in Sciences and Technologies of Engineering and Health (STIS), ENSIAS, Mohammed V University, \\ Rabat, Morocco \\ Correspondence should be addressed to Abdiddaim Katkout; abdiddaim.katkout@um5s.net.ma
}

Received 11 March 2020; Revised 21 April 2020; Accepted 27 April 2020; Published 15 May 2020

Academic Editor: Haiyan Lu

Copyright (c) 2020 Abdiddaim Katkout et al. This is an open access article distributed under the Creative Commons Attribution License, which permits unrestricted use, distribution, and reproduction in any medium, provided the original work is properly cited.

\begin{abstract}
The Three-Level Neutral-Point-Clamped (3L-NPC) inverter fed Permanent Magnet Synchronous Motor (PMSM) drive is an attractive configuration for high performance Electric Vehicle (EV) applications. For such configuration, due to their high performances, the Finite-Control-Set Model Predictive Control (FCS-MPC) is a very attractive control solution. The FCS-MPC scheme is based on the prediction of the future behavior of the controlled variables using the dynamic model of PMSM and the discrete nature of the 3L-NPC inverter. However, the parametric uncertainties and time-varying parameters affect the FCS-MPC algorithm performances. In this paper, robust FCS-MPC controls based on "dynamic error correction" (DEC) and "modified revised prediction" (MRP) are proposed to improve the FCS-MPC robustness without affecting the controller performances and complexity. The proposed strategies are improved also by multiobjective (MO) algorithm optimization and computation delay compensation. The simulation results included prove the performance in robustness and efficiency of the proposed robust FCSMPC-DEC.
\end{abstract}

\section{Introduction}

The Electric Vehicle (EV) has received more attention as an alternative solution of energy crisis and environmental challenges [1]. In the next years, with the research efforts and the constant improvement of the electrical drive systems and control strategies, the penetration of EVs will increase exponentially [2]. The electrical drive part of EVs is constituted by electric machine, power converter, and drive train [3]. Currently, a great variety of electrical drive technologies can be used on EVs: two/multilevel power converter and induction machines (IM)/Permanent Magnet Synchronous Motor (PMSM) electric machine. For the electric machine part, the PMSM is considered as the potential candidates for EVs applications with potential high energy density, high efficiency, easier control scheme, robust operations, and reduced maintenance [4]. Generally, the two-level inverters are widely used to control the PMSM in EVs applications [5]. To deal with this structure, several control strategies are proposed: linear controls with the modulator (e.g., fieldoriented control and direct torque control) and nonlinear controls without the modulator (e.g., switching table based) [6]. In different aspects, other classification include adaptive control (AC), sliding mode control (SMC), and active disturbance rejection control (ADRC) [7-10]. In the recent years, many Model Predictive Control (MPC) approaches are developed to improve the control performances for the power converter applications [11]. Based on the mathematical system model, MPC predict the future behavior of the system. The optimal control is obtained using predefined optimization criteria. The MPC presents several advantages: simple to apply in variety of processes and multiple-input- 
multiple-output (MIMO) systems, the system constraints and nonlinearities are considered in the control system. Furthermore, several control loops can be incorporated into one control law. Particularly, the Finite-Control-Set Model Predictive Control (FCS-MPC) is considered as attractive alternative predictive control [12]. Taking into account the discrete nature of the power converter, FSC-MPC uses the possible output voltage vectors to formulate the control algorithm and not require any external modulator stage. Furthermore, FCS-MPC provides good dynamic-state and steady-state performances [13]. In EVs' applications, research efforts have been made to improve the efficiency of FCS-MPC algorithms. Due to the parameter variation and road conditions, the EVs' process is considered as a timevarying system. The dynamic behavior of EVs, model uncertainties and inexactitude, unmodelled dynamics (motor and inverter), and unmeasurable parameters lead to a mismatch between the actual parameter values and their nominal values used in the prediction model and degrade the control robustness. There are many research studies accorded to robustness of the MPC controller [14]. A novelsimplified MPC scheme combined with the Lyapunov-based concept for interior PMSM is proposed in [15] to eliminate the flux-current mapping from the MPC formulation. Stumper et al. [15] propose a robust extension for predictive torque control (PTC) to improve the steady-state performances against parametric uncertainties for PMSM based on two-degree-of-freedom (2DoF) control. Then, the switching frequency and the ripples are eliminated without affecting the dynamics-state performances. In [16], the authors design a new discrete-time robust predictive current controller for PMSM. Using the static error correction (SEC) principle, the robustness of predictive current control of PMSM against the effect of the inaccurate of motor behavior is improved and implemented by a DSP model TMS320F2812 practically [17]. Nevertheless, the two-level inverters limit the set of control in eight possible combinations which lead to high output current/voltage total harmonic distortion (THD) and high switching losses. Furthermore, a high dv/dt derivative causes high stress in the semiconductor devices. To overcome these challenges, in the last few years, several multilevel inverter structures are recognized as a novel approach of high power converter performances [18]. Among them, the three-level neutral-point-clamped (3L-NPC) is the most interesting multilevel converter topology due to economy, complexity of control, and performances [19]. However, the computational burden will increase exponentially with the level of the power converter. This issue is addressed in [20] and the computational burden is reduced with $12.31 \%$. The robustness of the FCS-MPC control for the multilevel converter fed PMSM high performances (e.g., EVs' applications) is still an open research topic. In [6], based on onehorizon simple revised prediction (SRP), the system robustness against parameters variations is improved by reducing the control variables ripples. The SRP principle has been implemented on a fully FPGA-based platform (NIcRIO 9082 system). Typically, the existing research works are based on mono-objective problem optimization, without computational delay compensation, and adopted for two- level power converters. The main contributions in this work are as follows:

(i) Improved the predictive control robustness against parameters variation and uncertainties using "dynamic error correction" (DEC) and "modified revised prediction" (MRP) principles without affecting the system complexity and control dynamic-state performances.

(ii) A multiobjective problem optimization: current tracking control, neutral-point voltage control, common-mode voltage control, and reduction of switching frequency. These control objectives are controlled using single loop and one control action without internal/external loops and additional tables.

The main abovementioned contributions are adopted by the multilevel power converters structures with computational delay compensation based on the proposed modified two-step horizon of prediction approach.

The remainder of the paper is structured as follows. The problem statement is given in Section 2. The three principle elements in the MPC strategies are discussed. The prediction model is stated in Section 3. Cost function and optimization algorithm are illustrated in Section 4. In Section 5, the robustness of the proposed algorithms is verified by simulation using different scenarios.

\section{Problem Statement}

The MPC strategy is based on the explicit use of the system model to select the optimal action $s_{a b c}^{\text {opt }}=\left\{s_{a}, s_{b}, s_{c}\right\}$. Then, the performances of the MPC controller depend on the accuracy of the system model and its parameters. In this section, the effects of the variable system uncertainties, computational delay, and standard FCS-MPC principle are presented.

2.1. Impact of Variable System Uncertainties. The MPC controller established the optimal control action based on information about the dynamic behavior of the system. The system performances are affected with the accuracy of the information about the inductance $L_{d q}$ (time-varying variable) and the permanent magnet flux linkage $\psi_{p m}$ (unmeasurable variable). A variation in $\psi_{p m}$ value only affects the feed-through matrix, $H_{d}(k)$; on the contrary, a variation in $L_{d q}$ value affects both the system and control matrices, $A_{d}(k)$ and $B_{d}$ (equation (15)).

2.2. Impact of Computational Delay. In the ideal case, due to negligibility of the calculation time, the optimal control action $s_{a b c}^{\mathrm{opt}}$ that minimizes the cost function $f$ at instant $k+1$ is applied at time $k$. Consequently, the controlled variable reaches their predicted value at $k+1$ time. On the contrary, in the real case, a significant calculation time $t_{k}$ is required between the instants $k$ when the system state $x_{m}(k)$ is measured and the generation of the optimal action $s_{a b c}^{\mathrm{opt}}$ at $k+t_{k}$. The computational delay affects the system 
performances and lead to the oscillation of the controlled variables around their references values [21].

2.3. Standard FCS-MPC. In this work, four control objectives (COs) are considered:

(i) (CO1) Fast and accurate reference current tracking to reduce the mechanical component stress of system and acoustic pollution

(ii) (CO2) Capacitor voltage balancing between upper and lower DC-link capacitors to reduce the sensibility of output inverter voltage to the ripples in DClink voltage

(iii) (CO3) Limit the peak of common-mode voltage to reduce the overvoltage stress and increase the safety and reliability of electrical machine part of EVs

(iv) (CO4) Minimize the switching frequency to reduce the switching losses

These control objectives are incorporated in a single cost function and can be designed as follows:

$$
\begin{aligned}
f & =f_{i}+f_{D C}+f_{c m v}+f_{N c}, \\
f_{i} & =\lambda_{i}\left|i^{\text {ref }}(k+1)-i^{p}(k+1)\right|, \\
f_{D C} & =\lambda_{D C}\left|u_{z}^{p}(k+1)\right|, \\
f_{c m v} & =\lambda_{c m v}\left|u_{c m v}^{p}(k+1)\right|, \\
f_{N c} & =\lambda_{N c} N_{c},
\end{aligned}
$$

where $f_{i}, f_{D C}, f_{c m v}$, and $f_{N c}$ are the cost functions for current tracking control, neutral-point voltage control, common-mode voltage control, and reduction of switching frequency, respectively. $i^{\text {ref }}(k+1)$ and $i^{p}(k+1)$ represent the reference and predicted currents at instant $k+1$, respectively. $u_{z}^{p}(k+1)$ and $u_{c m v}^{p}(k+1)$ denote predicted neutral point and common-mode voltages, respectively. $\lambda_{i}$, $\lambda_{D C}, \lambda_{c m v}$, and $\lambda_{N c}$ are the weightings factors. It is worth mentioning that the weighting factors values have a direct influence on the controlled variables. The good performances of multiobjective optimization-based predictive control are guaranteed by the choice of the optimal weighting factors. In this work, the weighting factors $\lambda_{i}, \lambda_{D C}$, $\lambda_{c m v}$, and $\lambda_{N c}$ are tuned using "trial-and-error" manner.

In the cost function (5), $N_{c}$ penalize the switching state variation as follows:

$$
N_{c}=\sum_{x=a, b, c}\left|s_{x}(k+1)-s_{x}(k)\right| .
$$

\section{System Dynamic}

3.1. PMSM. The dynamic model of PMSM in the $d-q$ rotating reference frame is given by the differential equation [22]:

$$
\begin{aligned}
& \frac{\mathrm{d} i_{d}}{\mathrm{~d} t}=-\frac{R_{s}}{L_{d}} i_{d}(t)+\frac{L_{q}}{L_{d}} \omega_{e}(t) i_{q}(t)+\frac{1}{L_{d}} u_{s}^{d}(t), \\
& \frac{\mathrm{d} i_{q}}{\mathrm{~d} t}=-\frac{R_{s}}{L_{q}} i_{q}(t)+\frac{L_{d}}{L_{q}} \omega_{e}(t) i_{d}(t)-\frac{1}{L_{q}} \omega_{e}(t) \psi_{p m}+\frac{1}{L_{d}} u_{s}^{q}(t),
\end{aligned}
$$

$\frac{\omega_{e}(t)}{\mathrm{d} t}=\frac{Z_{p}}{J_{m}} T_{e}-\frac{B_{v}}{J_{m}} \omega_{e}(t)-\frac{Z_{p}}{J_{m}} T_{L}$,

where $u_{s}^{d q}$ is stator $d q$-voltages, $\mathrm{V} ; i^{d q}$ is stator $d q$-currents, A; $R_{s}$ is stator winding resistance, $\Omega ; L_{d}$ and $L_{q}$ are stator $d q$ inductances, $\mathrm{H} ; \omega_{e}$ is electric rotatory frequency, $\mathrm{rad} / \mathrm{s} ; \psi_{p m}$ is permanent magnet flux, $\mathrm{Wb} ; T_{e}$ and $T_{L}$ electromagnetic and load torques, Nm; $Z_{p}$ is the number of pole pairs; $I_{m}$ is moment of inertia, $\mathrm{kg} . \mathrm{m}^{2} ; B_{v}$ is friction coefficient, Nm.s; and $T_{s}$ sampling interval, $s$. The electromagnetic torque of PMSM is obtained by [23]

$$
T_{e}=\frac{3}{2} Z_{p}\left(\psi_{p m} i_{q}(t)+\left(L_{d}-L_{q}\right) i_{d}(t) i_{q}(t)\right) .
$$

Remark 1. Due to the uniform air-gap in surface mounted PMSM (SPMSM), the stator $d q$-inductance are identical to each other: $L_{d}=L_{q}=L_{s}$. Opposed to SPMSM, the internal PMSM (IPMSM) is specified by the nonuniform air-gap flux. Thus, the stator $q$-inductance $L_{q}$ is smaller than stator $d$ inductance $L_{d}$. The approximation $L_{d}=L_{q}=L_{s}$ of SPMSM is considered in the rest of the paper.

Using the $d q$-current dynamic and the switching state of 3L-NPC inverter, the neutral-point voltage dynamic is [24]

$$
\frac{\mathrm{d} u_{z}}{\mathrm{~d} t}=\frac{1}{4 C}\left(\left(2\left|s_{a}\right|-\left|s_{b}\right|-\left|s_{c}\right|\right) i_{d}+\sqrt{3}\left(\left|s_{b}\right|-\left|s_{c}\right|\right) i_{q}\right),
$$

where $C=C_{1}=C_{2}$ is the DC-link capacitor (Figure 1(a)).

3.2. $3 L-N P C$ Inverter. The $3 \mathrm{~L}-\mathrm{NPC}$ inverter consists of three identical branches, each branch is composed with four switches and can be described by three switching states [1], $[0]$, and $[-1]$ (Table 1$)$. Thus, there are 27 possible combinations of switching states corresponding to 19 inverter output voltages obtained in $\alpha \beta$-coordinate as follows [20]:

$$
u_{s}^{\alpha \beta}=U_{D C}\left(\left(2 s_{a}-s_{b}-s_{c}\right)+j \sqrt{3}\left(s_{b}-s_{c}\right)\right),
$$

where $U_{D C}$ denotes the DC-link voltage.

In $a b c$ coordinate, the common-mode voltage $u_{C M V}$ generated by the $3 \mathrm{~L}-\mathrm{NPC}$ inverter can be presented by [24]

$$
u_{C M V}=\frac{u_{A Z}-u_{B Z}-u_{C Z}}{3} .
$$

$u_{A Z}$ is defined as the voltage between the phase (A) and the neutral-point voltage (Z) (Figure 1(a)). Table 2 shows the switching states and corresponding $u_{C M V}$ [25]. Simplified electrical circuit of 3L-NPC inverter-fed PMSM drives are presented in Figure 1(a). 


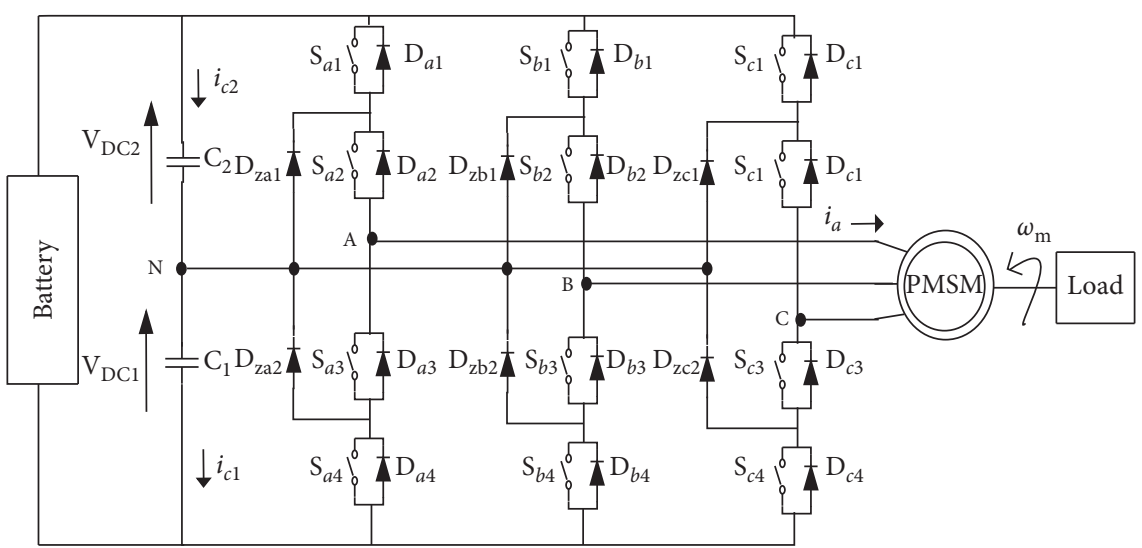

(a)

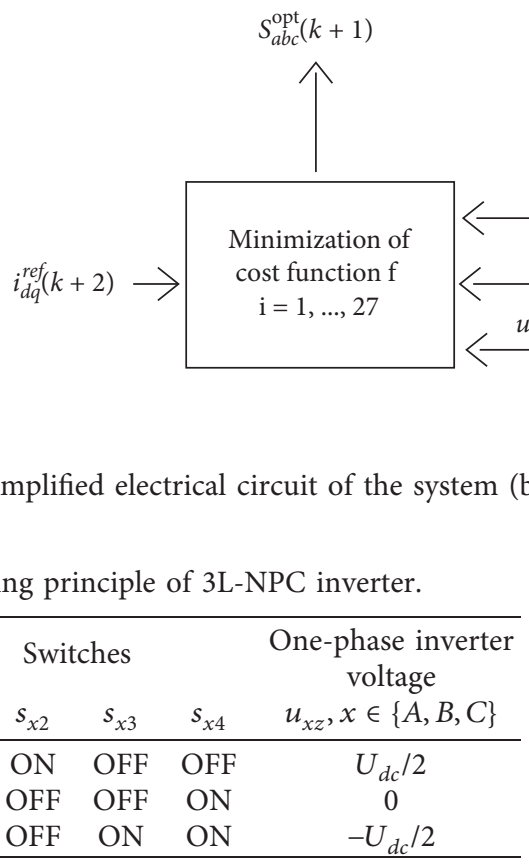

TABLE 2: $u_{C M V}$ corresponding for every switching state combination.

\begin{tabular}{lc}
\hline Switching state combination & $u_{c m v}$ \\
\hline 111 & $U_{d c} / 2$ \\
$-1-1-1$ & $-U_{d c} / 2$ \\
$110,011,101$ & $U_{d c} / 3$ \\
$0-1-1,-10-1,-1-10$ & $-U_{d c} / 3$ \\
$100,010,001,11-1,-111,1-11$ & $U_{d c} / 6$ \\
$00-1,-100,0-10,1-1-1,-11-1,-1-11$ & $-U_{d c} / 6$ \\
$000,10-1,01-1,-110,-1010-11,1-10$ & 0 \\
\hline
\end{tabular}

\section{FCS-MPCs Algorithms}

4.1. Standard FCS-MPC with Improved Cost Function (FCS$M P C-I C F)$. Considering a sampling period $T_{s}$, the discrete state space model of PMSM is obtained by employing the forward Euler method for equations (7) and (8):

$$
x_{m}(k+1)=A_{d}(k) x_{m}(k)+B_{d} u_{m}(k)+H_{d}(k),
$$

where $x_{m}(k)=\left[\begin{array}{ll}i_{m}^{d} & i_{m}^{q}\end{array}\right]^{T}$ and $u_{m}(k)=\left[\begin{array}{ll}u_{s}^{d} & u_{s}^{q}\end{array}\right]^{T} . A_{d}(k)$, $B_{d}$, and $H_{d}(k)$ are the system, input, and feed-through matrices. They are given by

$$
\begin{aligned}
A_{d}(k) & =\left[\begin{array}{cc}
1-\frac{R_{s} T_{s}}{L_{s}} & T_{s} \omega_{e}(k) \\
-T_{s} \omega_{e}(k) & 1-\frac{R_{s} T_{s}}{L_{s}}
\end{array}\right], \\
B_{d} & =\left[\begin{array}{cc}
\frac{T_{s}}{L_{s}} & 0 \\
0 & \frac{T_{s}}{L_{s}}
\end{array}\right], \\
H_{d}(k)= & {\left[\begin{array}{c}
T_{s} \psi_{p m}(k) \\
-\frac{L_{s}}{2} \omega_{e}(k)
\end{array}\right] . }
\end{aligned}
$$

The predictive reference current is calculated using the Lagrange method [20]:

$$
i^{\mathrm{ref}}(k+1)=3 i^{\mathrm{ref}}(k)-3 i^{\mathrm{ref}}(k-1)+i^{\mathrm{ref}}(k-2) .
$$

The evolution of neutral-point voltage is defined as follows: 


$$
\begin{aligned}
u_{z}^{p}(k+1)= & u_{z}(k)+\frac{T_{s}}{4 C}\left(2\left|s_{a}(k)\right|-\left|s_{b}(k)\right|-\left|s_{c}(k)\right|\right) i_{\alpha}(k) \\
& +\frac{\sqrt{3} T_{s}}{4 C}\left(2\left|s_{b}(k)\right|-\left|s_{c}(k)\right|\right) i_{\beta}(k) .
\end{aligned}
$$

To compensate the computational delay, according to long horizon of prediction approach, we propose to use two-step horizon of prediction. In the 3L-NPC inverter case, combination of 27 switching states are available, a discrete set of $27^{2}=729$ possible trajectories of the switching states is required to evaluate the cost function led to a large number of cost computations which makes the real challenge to the actual digital system implementation. To reduce the number of real time evaluation, this paper proposes to use a modified two-step horizon prediction by applying the same voltage vector in the twostep horizon. The improved cost function $f^{\mathrm{dcom}}$ is evaluated at time $k+2$ to generate the optimal switching state at time $k+1$ :

$$
\begin{aligned}
& f^{\mathrm{dcom}}=f_{i}^{\mathrm{dcom}}+f_{D C}^{\mathrm{dcom}}+f_{c m v}^{\mathrm{dcom}}+f_{N c}^{\mathrm{dcom}}, \\
& f_{i}^{\mathrm{dcom}}=\lambda_{i}\left|i^{\mathrm{ref}}(k+1)-i^{p}(k+1)\right|, \\
& f_{D C}^{\mathrm{dcom}}=\lambda_{D C}\left|u_{z}^{p}(k+1)\right|, \\
& f_{c m v}^{\mathrm{dcom}}=\lambda_{c m v}\left|u_{c m v}^{p}(k+1)\right|, \\
& f_{N c}^{\mathrm{dcom}}=\lambda_{N c} N_{c}^{\mathrm{dcom}}, \\
& N_{c}^{\mathrm{dcom}}=\sum_{x=a, b, c}\left|s_{x}(k+2)-s_{x}(k)\right| .
\end{aligned}
$$

The reference current $i^{\text {ref }}(k+2)$ is extrapolated by using the second-order Lagrange extrapolation [20]:

$$
i^{\mathrm{ref}}(k+2)=6 i^{\mathrm{ref}}(k)-8 i^{\mathrm{ref}}(k-1)+3 i^{\mathrm{ref}}(k-2) .
$$

The evolution of $x_{m}$ and $u_{z}^{p}$ at instant $k+2$ are expressed based on equations (14) and (17):

$$
\begin{aligned}
x_{m}(k+2)= & A_{d}(k+1) x_{m}(k+1)+B_{d} u_{m}(k+1) \\
& +H_{d} v(k+1), \\
u_{z}^{p}(k+2)= & u_{z}(k+1)+\frac{T_{s}}{4 C}\left(2\left|S_{a}(k+1)\right|-\left|S_{b}(k+1)\right|\right. \\
& \left.-\left|S_{c}(k+1)\right|\right) i_{\alpha}(k+1)+\frac{\sqrt{3} T_{s}}{4 C}\left(2\left|S_{b}(k+1)\right|\right. \\
& \left.-\left|S_{c}(k+1)\right|\right) i_{\beta}(k+1) .
\end{aligned}
$$

In order to guarantee the stability, a Lyapunov candidate function (LCF) can be used to ensure that the stator current $i_{d q}$ are maintained according to their reference value $i_{d q}^{\text {ref }}$ as follows [26]:

$$
V\left(\widetilde{i}_{d}, \widetilde{i}_{q}\right)=\frac{1}{2} \Upsilon_{d} \tilde{i}_{d}^{2}+\frac{1}{2} \Upsilon_{q} \widetilde{i}_{q}^{2} .
$$

With

$$
\Delta V=V\left(\widetilde{i}_{d}^{p}(k+1), \tilde{i}_{q}^{p}(k+1)\right)-V\left(\widetilde{i}_{d}(k), \widetilde{i}_{q}(k)\right),
$$

where $\Upsilon_{d}$ and $\Upsilon_{q}$ are the positive gains, $\tilde{i}_{d q}=\left[\widetilde{i}_{d}, \widetilde{i}_{q}\right]$ is the state error of the stator current defined as follows:

$$
\widetilde{i}_{d q}=\left[\begin{array}{c}
i_{d}^{m}(k) \\
i_{q}^{m}(k)
\end{array}\right]-\left[\begin{array}{c}
i_{d}^{\mathrm{ref}}(k) \\
i_{q}^{\mathrm{ref}}(k)
\end{array}\right] .
$$

The optimal switching state $s_{a b c}^{\mathrm{opt}}$ is given by the following equation:

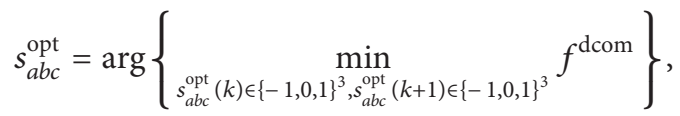

$$
\begin{gathered}
\text { subject to }\left(\left(V(k)>\varrho_{v}\right) \&(\Delta V<0)\right) \vee\left(\left(V(k)<\varrho_{v}\right) \&\right. \\
\left.\cdot\left(V(k+1)<\varrho_{v}\right)\right) \\
s_{a b c}(k)=s_{a b c}(k+1) .
\end{gathered}
$$

With the aim to ensure the stability of the closed loop, two cases are considered [26]: the first one corresponds to $V(k)>\varrho_{v}$ and the second one $V(k)<\varrho_{v}$. If $V(k)>\varrho_{v}$ only switches obtaining $\Delta V<0$ are considered and if $V(k)>\varrho_{v}$ only switches assuring $V(k+1)<\varrho_{v}$ are considered. Hence, $\varrho_{v}$ can be used as a stabilizing constraint, which guarantees feasibility and stability for the optimization problem.

Any parameters variation or uncertainties compensation is included in the standard FCS-MPC-ICF algorithm control formulation. A simplified block diagram of standard FCSMPC-ICF is presented in Figure 1(b). It is worth to be mentioned that the "error correction" and "revised prediction" principles are based on the same approach: modified prediction equation to take into account the time-varying behavior of the system. EC is based on the contribution of the controlled parameter error in the previous sampling period in the formulation of optimization problem. On the contrary, RP improves the robustness against the variations of controlled variables taking their different roles into account. These principles are developed in this work based on "dynamic error correction" and "modified revised prediction," and they are implemented separately in robust FCS-MPC-DEC and robust FCS-MPC-MRP controls. In both proposed robust predictive controls, the parameters variation and uncertainties are explicitly included in the algorithms formulation.

4.2. Robust FCS-MPC-DEC. The future behavior of the controlled system is predicted using equation (12) for the 27 possible combinations in the $3 \mathrm{~L}-\mathrm{NPC}$ inverter for each prediction horizon. The accuracy of the prediction is directly affected by the quality of the parameter information. The EC 


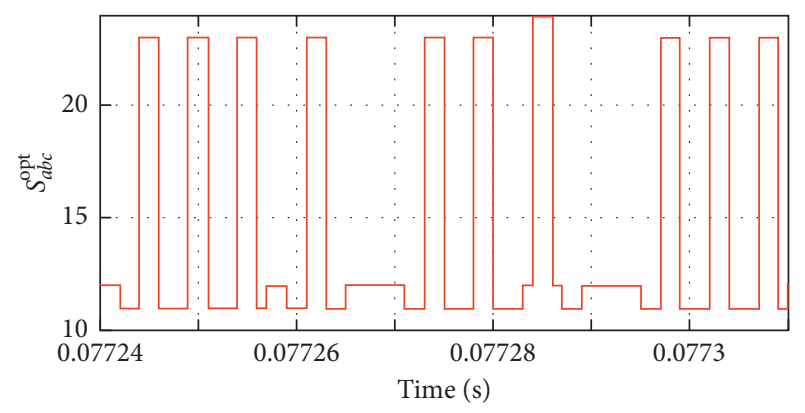

(a)

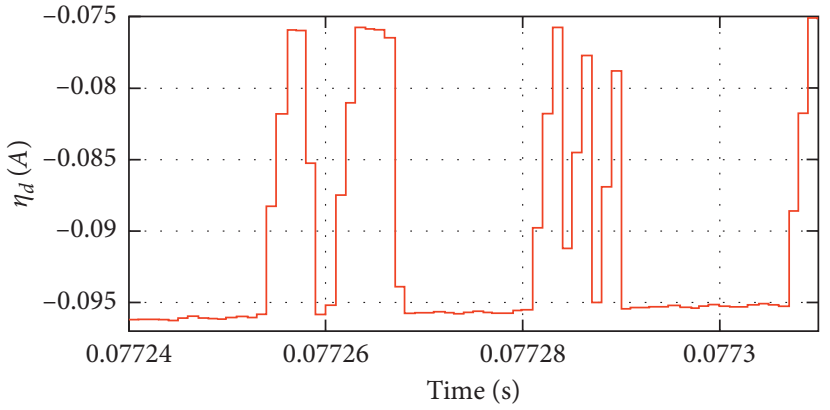

(b)

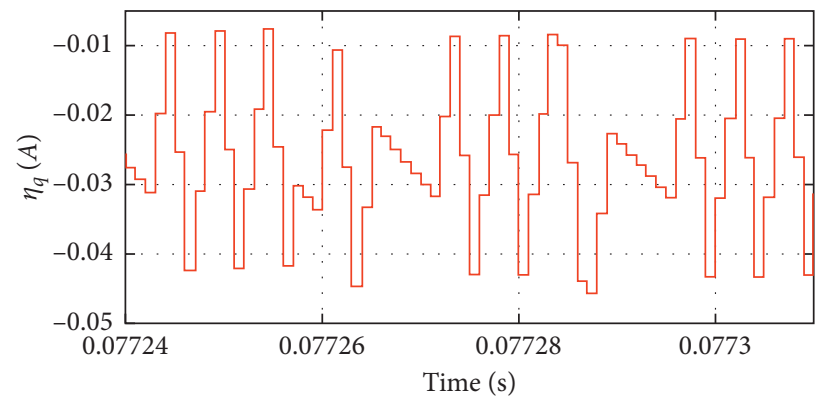

(c)

Figure 2: Behavior of standard FCS-MPC-ICF at normal conditions. (a) The stator current $i_{a}$. (b) The output voltage $U_{a}$. (c) Fast Fourier Transform of the current. (d) Fast Fourier Transform of the output voltage. (e) Performance of the neutral-point voltage $U_{z}$. (f) Performance of common-mode voltage $U_{c m v}$.

is based on the contribution of the weighted error between the predicted and measured controlled variable values in the previous sampling time $\eta_{d q}(k-1)$, in the prediction equation. In [15], the author used the previous current error without considering the optimal switching state combination applied in the next sampling interval. Due to the variation of the switching state applied in every sampling time, the direct consideration of the previous error is not suitable (Figure 2). Siami et al. [17] proposed to use a static weighted error for each switching state combination $j(j=1$, .., 8). In this study, we proposed to use a dynamic DEC for every given switching combinations $j(j=1, \ldots, 27)$. The weighted error is calculated for each possible switching state combination. The modified predicted state is

$$
i_{d q, j}^{p, m o d}(k+1)=i_{d q, j}^{p}(k+1)+\sigma_{1} \eta_{d q, j}(k),
$$

where $\sigma_{1}$ is a weighting factor between 0 and 1 and $\eta_{d q, j}$ is the discrete current error defined as follows:

$$
\eta_{d q, j}(k)=\left[\begin{array}{c}
i_{d, j}^{p}(k) \\
i_{q, j}^{p}(k)
\end{array}\right]-\left[\begin{array}{c}
i_{d, j}^{m}(k) \\
i_{q, j}^{m}(k)
\end{array}\right] .
$$

\subsection{Robust FCS-MPC-MRP}

4.3.1. Robustness against $L_{s}$ Variation. To consider the mismatch between the actual value of the inductance and their nominal value used in the controller, the system state is modified as follows:

$$
x_{m}^{\bmod }(k)=\sigma_{2} x_{m}(k)+\left(1-\sigma_{2}\right) x_{p}(k),
$$

where $x_{m}$ and $x_{p}$ are the measured and predicted values of the system state, respectively, $\sigma_{2}$ denotes a weighting factor between 0 and 1 according to the BIBO stability criteria (in Appendix). The tunable parameter will add a "weighted average" process to the measured and predicted values of the system. Therefore, the modified state of $x_{m}^{\text {mod }}$, which takes the benefits of this ("weighted average" process), will also add a smoothing and filtering effect to the system states whenever with or without parameter variations. Consequently, improved control variable quality is predictable.

4.3.2. Robustness against $\psi_{p m}$ Variation. Introducing $L_{s}=$ $L_{s 0}+\Delta L_{s}$ and $\psi_{p m}=\psi_{p m 0}+\Delta \psi_{p m}$, where $L_{s 0}$ and $\psi_{p m 0}$ are the nominal values and $\Delta L_{s}$ and $\Delta \psi_{p m}$ are the parametric uncertainties, and using equation (14), the relationship between the measured and the predicted current values is

$$
\begin{aligned}
i_{q}^{m}(k)= & \frac{L_{s 0}}{L_{s}} i_{q}^{m}(k)+\frac{\Delta L_{s}}{L_{s}} T_{s} \omega_{e}(k-1) i_{d}^{p}(k-1) \\
& +\frac{1}{L_{s}} T_{s} \omega_{e}(k-1) \Delta \psi_{p m} .
\end{aligned}
$$

In the steady-state SPMSM operation $\left(i_{d}=0\right)$, the error between the predicted and measured $q$-axis current is 
Read $i_{d q}^{\text {ref }}(k)$ and extrapolate $i_{d q}^{\text {ref }}=(k+1)$ and $i_{d q}^{\text {ref }}(k+2)$ by using equations (16) and (24);

Measure $i_{d q}^{m}(k), \omega_{e}(k)$ and $u_{z}(k)$;

$\%$ Initialize the values of $s_{a b c}^{\text {opt }}$ and $f^{\text {dcom }}$

$s_{a b c}^{\mathrm{opt}}=1 ; f^{\mathrm{dcom}}=\operatorname{zeros}(1: 27)$;

For $i=1$ to 27 do

Calculate $u_{s}^{\alpha \beta}$ based on equation (12);

Predict $i_{d q}^{p}(k+1)$ and $i_{d q}^{p}(k+2)$ from equations (14) and (25);

Evaluate the candidate Lyapunov function: $V(k+1), V(k), \Delta V$ using equations (27) and (28);

if $\left(\left(V(k)>\epsilon_{v}\right) \&(\Delta V<0)\right) \vee\left(\left(V(k)<\epsilon_{v}\right) \&\left(V(k+1)<\epsilon_{v}\right)\right)$ then

Predict $u_{z}^{p}(k+1)$ and $u_{z}^{p}(k+2)$ based on equations (17) and (26);

Calculated $u_{c m v}^{p}(i)$ from Table 2 ;

Calculate $N_{c}^{\text {dcom }}$ from equation (23);

Compute the cost functions $f_{i}^{\mathrm{dcom}}, f_{D C}^{\mathrm{dcom}}, f_{c m v}^{\mathrm{dcom}}$ and $f_{N c}^{\mathrm{dcom}}$ based on equations (19)-(22);

Evaluate the cost function $f^{\mathrm{dcom}}$ by using equation (18);

End

End

Select and applied the optimal switching state $s_{a b c}^{\mathrm{opt}}=\min \left(f^{\mathrm{dcom}}\right)$;

Algorithm 1: Standard FCS-MPC-ICF.

$$
\eta_{q}^{m}(k)=i_{q}^{m}(k)-i_{q}^{p}(k) \approx \frac{1}{L_{s}} T_{s} \omega_{e}(k-1) \Delta \psi_{p m} .
$$

The permanent magnet flux uncertainties will contribute into the current error. To compensate the effect of the permanent magnet flux uncertainties into the equation prediction equation (35), a modified permanent magnet flux is proposed as follows:

$$
\psi_{p m}^{\bmod }(k)=\psi_{p m}(k)+\sigma_{3} \sum\left(\eta_{q}^{m}(k)\right)
$$

where $\sigma_{3}$ is the weighted factor.

The proposed control algorithm for standard FCSMPC-ICF, robust FCS-MPC-DEC, and robust FCS-MPCMRP are described in Algorithms 1-3, respectively. Algorithm 1 shows the detailed instructions, equations, and parameters used in the simulation of standard FCS-MPCICF. The algorithms 1 and 2 can be summarized in the following steps: measurements, calculation, and extrapolation of references and prediction of control variables and minimization of the improved cost function. Both Algorithms 3 and 4 illustrated the specific instructions and parameters used in proposed robust FCS-MPC-DEC and robust FCS-MPC-MRP (e.g., modified system state and current error vector).

Remark 2. The factors $\sigma_{1}, \sigma_{2}$, and $\sigma_{3}$ are tuned in a "trialand-error" manner.

\section{Results and Analysis}

In this section, the control performances of proposed robust FCS-MPC-DEC and robust FCS-MPC-MRP for 3L-NPC inverter fed PMSM for EVs' applications are validated by simulations and compared with the control performance of standard FCS-MPC-ICF under different scenarios both with and without parameters variations and uncertainties. Using Matlab/Simulink software and SimPowerSystems toolbox, the simulations are carried with the parameters, as indicated in Table 3.

With the aim to study better the robustness of the proposed robust FCS-MPCs controls, we proposed to use the two performances variables.

(i) The mean absolute reference tracking error [21]:

$$
\bar{e}_{\text {MARTE }}(x)=\frac{1}{N} \sum_{i=1}^{N}\left|x_{\text {ref }}(i)-x_{\text {mes }}(i)\right| \text {. }
$$

$N$ denotes the number of simulation iterations. $x_{\text {ref }}$ and $x_{\text {mes }}$ are the reference and measured controlled variables $x$, respectively.

(ii) The average switching number per semiconductor:

$$
\bar{f}_{\mathrm{ASN}}=\frac{1}{N} \sum_{k=1}^{N} \frac{n_{i j}(k)}{12},
$$

where $n_{i j}$ is the switching numbers of power semiconductors of phase $i \in\{a, b, c\}$ and order $j \in\{1,2,3,4\}$ defined as follows:

$$
n_{i j}=\left|s_{i j}(k+1)-s_{i j}(k)\right| \text {. }
$$

The behavior of the standard FCS-MPC-ICF at the nominal conditions is illustrated in Figure 3. In the test, the good dynamic-state performances in fast and accurate reference current tracking are demonstrated (Figure 3(a)). The steady-state performances of the FCS-MPC-ICF are validated with low mean absolute reference tracking error and THD of current 0.0091 and 3.29 (Figure 3(c)), respectively. The output voltage is shown in Figure 3(b) with THD of 
$\%$ Initialize the values of $s_{a b c}^{\mathrm{opt}}, f^{\mathrm{dcom}}$ and current error $\eta_{d q, j}$;

$s_{a b c}^{\mathrm{opt}}=1 ; f^{\mathrm{dcom}}=\operatorname{zeros}(1: 27) ; \eta_{(d q, j)}=\operatorname{zeros}(2: 27)$;

For $i=1$ to 27 do

Calculate the current error $\eta_{(d q, j)(:, i)}$ based on equation (32);

Predict the modified $i_{d q, j}^{p, \bmod }(k+1)$ and $i_{d q, j}^{p, \bmod }(k+2)$ using the current error $\eta_{d q, j}(:, i)$ and equations (31) and (25);

End

Select and applied the optimal switching state $\left[\eta_{(d q, j)} s_{a b c}^{\mathrm{opt}}\right]=\min \left(f^{\mathrm{dcom}}\right)$;

Algorithm 2: Robust FCS-MPC-DEC.

$\%$ Initialize the values of $s_{a b c}^{\text {opt }}$ and $f^{\text {dcom }}$

$s_{a b c}^{\mathrm{opt}}=1 ; f^{\mathrm{dcom}}=\operatorname{zeros}(1: 27)$;

For $i=1$ to 27 do

Predict the modified $x_{m}^{\bmod }(k+1)$ and $x_{m}^{\bmod }(k+2)$ using equation (33) and (25);

End

Select and applied the optimal switching state $s_{a b c}^{\mathrm{opt}}=\min \left(f^{\mathrm{dcom}}\right)$;

Algorithm 3: Robust FCS-MPC-MRP against $L_{s}$ variation.

\% Initialize the values of $s_{a b c}^{\mathrm{opt}}, f^{\mathrm{dcom}}$ and $\sum \eta_{q}^{m}$

$s_{a b c}^{\mathrm{opt}}=1 ; f^{\mathrm{dcom}}=\operatorname{zeros}(1: 27) ; \sum \eta_{q}^{m}=0$;

For $i=1$ to 27 do

Calculate the current error $\eta_{q}^{m}$ and the summation $\sum \eta_{q}^{m}$ based on equation (36);

Calculate the modified permanent magnet flux $\psi_{p m}^{\text {mod }}$ using the equation (37);

End

Algorithm 4: Robust FCS-MPC-MRP against $\psi_{p m}$ variation.

TABLE 3: System parameters.

\begin{tabular}{lc}
\hline Parameter & Value \\
\hline PMSM power rate $P_{N}(\mathrm{~W})$ & 1440 \\
PMSM pole pairs $Z_{p}[1]$ & 2 \\
PMSM resistance $R_{s}[\Omega]$ & 2.89 \\
PMSM inductance $L_{s}(\mathrm{mH})$ & 7 \\
PM flux $\psi(\mathrm{Wb})$ & 0.125 \\
Moment of inertia $J_{m}\left[\mathrm{~kg} \cdot \mathrm{m}^{2}\right]$ & $10^{-5}$ \\
Friction coefficient $B_{v}(\mathrm{Nm} . \mathrm{s})$ & $11.10^{-5}$ \\
DC capacitance $C_{1}=C_{2}=C(\mu \mathrm{F})$ & 1 \\
\hline
\end{tabular}

22.79 (Figure 3(d)). The average switching number per semiconductor $\bar{f}_{\text {ASN }}$ is 9834 . As shown in Figure 3(e), the deviation of DC-link voltage $u_{z}$ is maintained around $6.66 \%$ of its nominal value. Figure 3(f) shows the evolution of $u_{c m v}$ and the overvoltage stresses of PMSM are reduced to an average value of 0.2 .

The evolution of current error $\eta_{d q}(k)$ in function of applied optimal switching state $s_{a b c}^{\mathrm{opt}}$ is illustrated in Figure 2.
The application of dynamic DEC is motivated by the dynamic behavior of $\eta_{d q}$ and its not constant for same $s_{a b c}^{\mathrm{opt}}$. Consequently, the proposition of fixed error values of each switching combination considered in static error correction is incorrect.

Figure 4 and Table 4 evaluates the robustness of standard FCS-MPC-ICF and proposed robust FCS-MPC-DEC and FCS-MPC-MRP controls against parameter system variations. The inductance $L_{s}$ and the permanent magnet flux $\psi_{p m}$ are varied to $-50 \%$ and $200 \%$ of their nominal values. The performances of standard FCS-MPC-ICF are affected in presence of variations of $L_{s}$ and $\psi_{p m}$, and the error and controlled variable ripples around their nominal values are highly increased. These control ripples run to the instability in case of high parameters variations (in case of $200 \%$ of parameter variation). In case of proposed robust FCS-MPCDEC and FCS-MPC-MRP, the robustness against parameter variation is improved. The proposed robust FCS-MPC-DEC solution outperforms both the proposed robust FCS-MPCMRP and standard FCS-MPC-ICF controls in terms of 


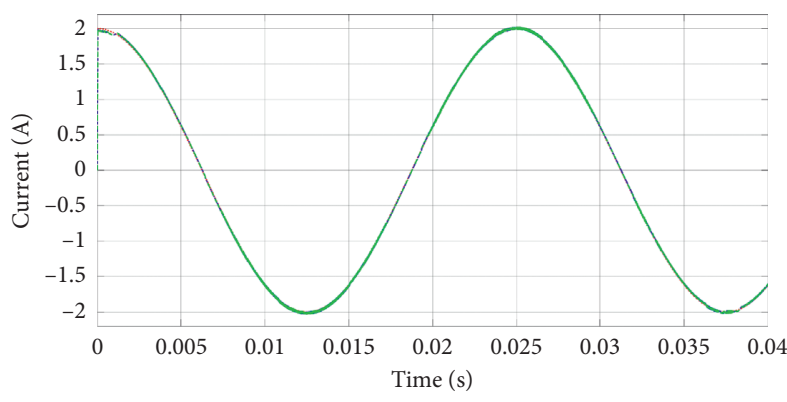

$-\begin{aligned} & \mathrm{i}_{\mathrm{a}}^{\mathrm{ref}} \\ & ---\mathrm{i}_{\mathrm{a}}^{\mathrm{m}} \\ & \ldots \ldots \cdot \mathrm{i}_{\mathrm{a}}^{\mathrm{p}}\end{aligned}$

(a)

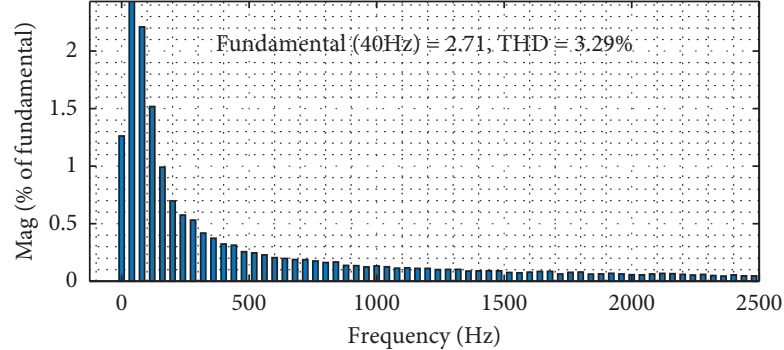

(c)

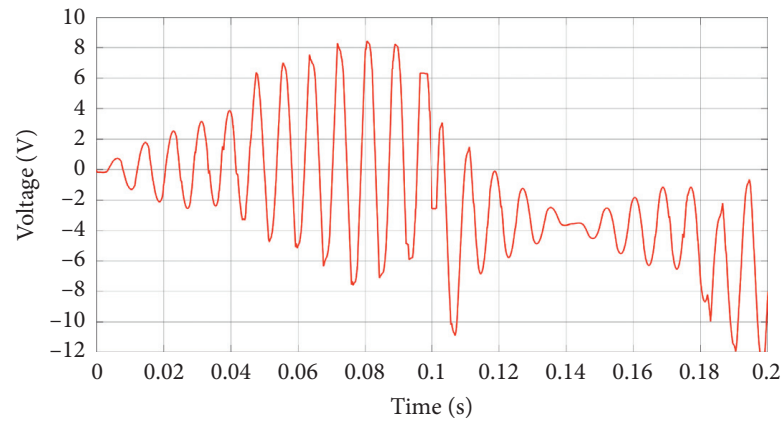

(e)

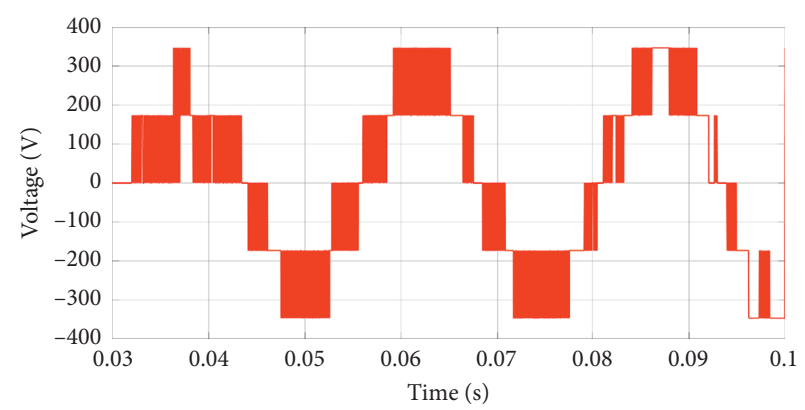

(b)

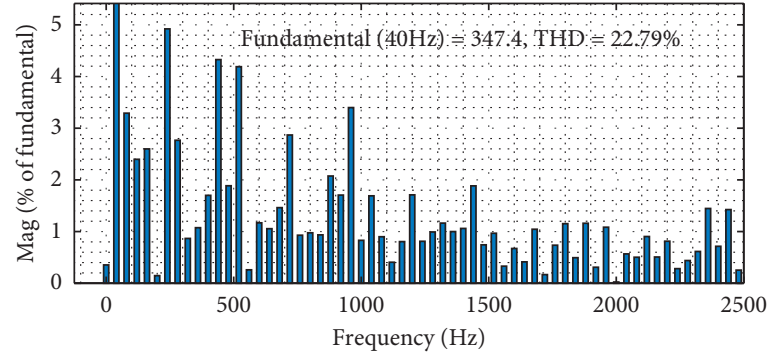

(d)

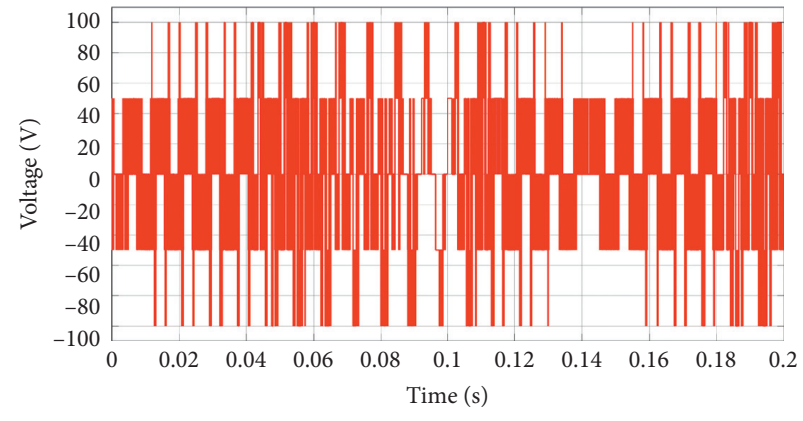

(f)

Figure 3: The evolution of current error $\eta_{d q}$ of standard FCS-MPC-ICF. (a) $s_{a b c}^{\mathrm{opt}}$. (b) The current error $\eta_{d}$. (c) The current error $\eta_{q}$.

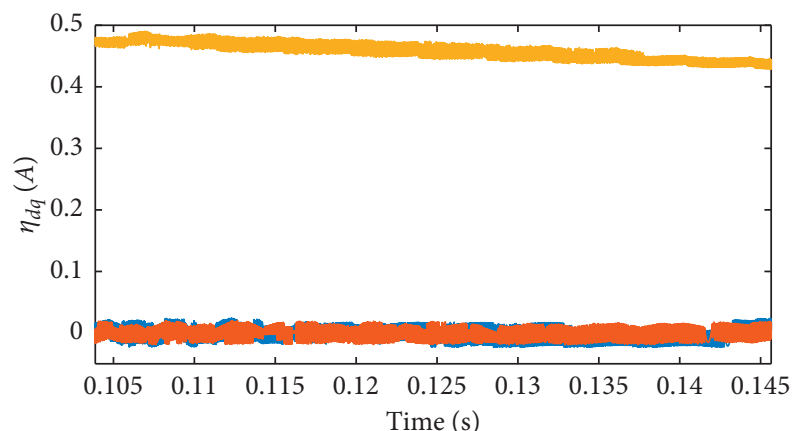

(a)

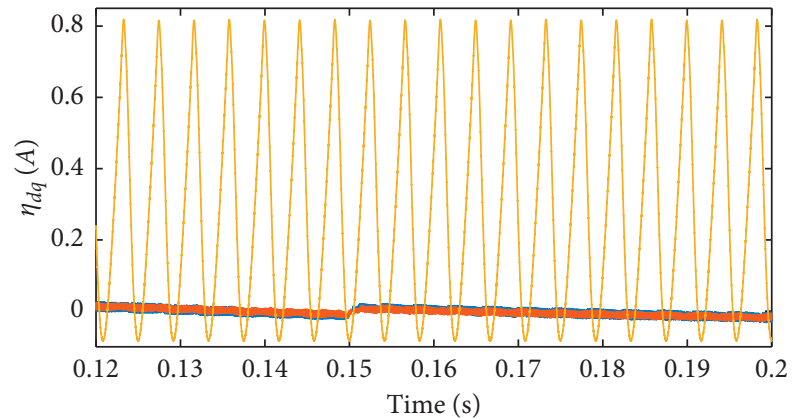

(b)

Figure 4: Continued. 


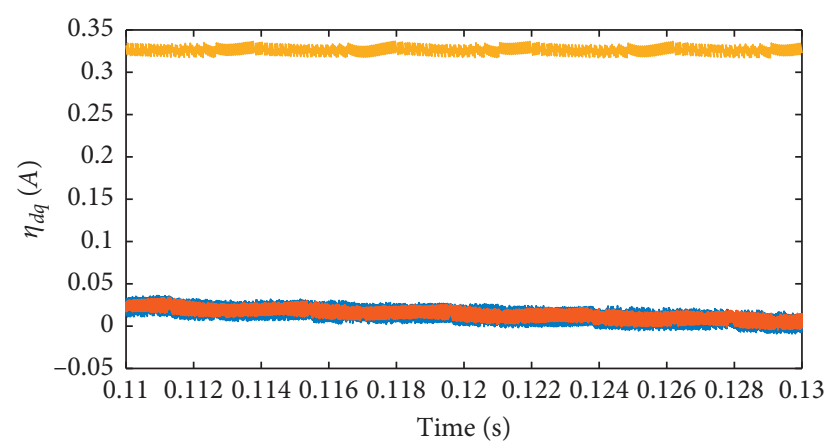

(c)

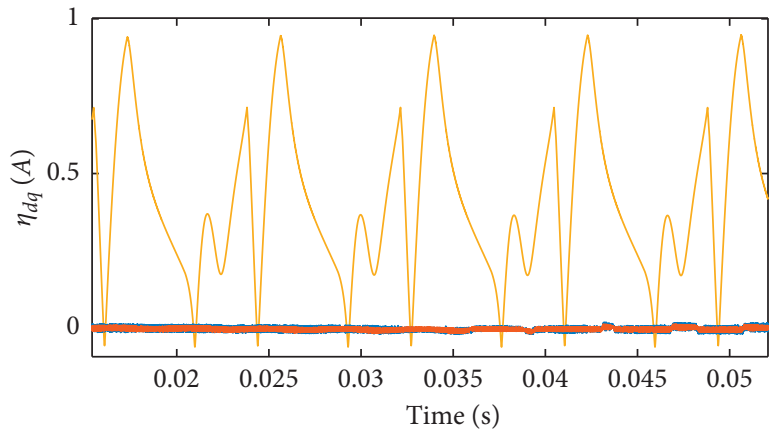

(d)

FIgURE 4: The evolution of current error $\eta_{d q}$ : (a) $0.5 L_{s}$, (b) $2 L_{s}$, (c) $0.5 \psi_{m g}$, and (d) $2 \psi_{m g}$; coral: standard FCS-MP-ICF, red: robust FCSMPC-DEC, and blue: robust FCS-MPC-MRP.

TABle 4: Comparison of the control performances under different controls.

\begin{tabular}{|c|c|c|c|c|c|c|c|c|c|}
\hline \multirow{2}{*}{ Parameter } & \multirow{2}{*}{ Variation } & \multirow{2}{*}{ Control } & \multirow{2}{*}{$\bar{e}_{\text {MARTE }}\left(i_{d q}\right)$} & \multirow[b]{2}{*}{$u_{c m v}$} & \multirow{2}{*}{$u_{z}(\%)$} & \multirow{2}{*}{$\bar{f}_{A S N}$} & \multirow{2}{*}{$t_{c}$} & \multicolumn{2}{|c|}{ THD } \\
\hline & & & & & & & & $i_{d q}$ & $u_{s}$ \\
\hline \multirow{6}{*}{$\Delta L_{s}$} & \multirow{3}{*}{$-50 \%$} & Standard FCS-MPC-ICF & 0.4892 & 0.76 & 7.05 & $1.6010^{4}$ & 21.96 & 4.63 & 29.06 \\
\hline & & Robust FCS-MPC-DEC & 0.0098 & 0.23 & 6.68 & $9.8310^{3}$ & 14.70 & 3.68 & 27.58 \\
\hline & & Robust FCS-MPC-MRP & 0.0100 & 0.25 & 6.79 & $1.2210^{4}$ & 18.80 & 3.73 & 28.26 \\
\hline & \multirow{3}{*}{$200 \%$} & Standard FCS-MPC-ICF & 0.5740 & 4.28 & 8.31 & $1.8510^{4}$ & 39.41 & 6.35 & 36.16 \\
\hline & & Robust FCS-MPC-DEC & 0.1297 & 0.75 & 7.16 & $4.1910^{3}$ & 31.57 & 4.68 & 35.23 \\
\hline & & Robust FCS-MPC-MRP & 0.1306 & 0.76 & 7.33 & $7.7110^{3}$ & 33.20 & 4.71 & 35.31 \\
\hline \multirow{6}{*}{$\Delta \psi_{p m}$} & \multirow{3}{*}{$-50 \%$} & Standard FCS-MPC-ICF & 0.3199 & 0.75 & 6.95 & $2.2810^{4}$ & 29.47 & 4.07 & 28.32 \\
\hline & & Robust FCS-MPC-DEC & 0.0124 & 0.28 & 6.81 & $1.9410^{3}$ & 25.66 & 3.45 & 25.70 \\
\hline & & Robust FCS-MPC-MRP & 0.0136 & 0.38 & 6.95 & $1.9410^{3}$ & 27.59 & 3.59 & 26.07 \\
\hline & \multirow{3}{*}{$200 \%$} & Standard FCS-MPC-ICF & 0.5037 & 2.43 & 8.23 & $2.3110^{4}$ & 47.80 & 6.01 & 70.84 \\
\hline & & Robust FCS-MPC-DEC & 0.0940 & 0.50 & 7.06 & $9.8310^{3}$ & 34.21 & 4.45 & 32.55 \\
\hline & & Robust FCS-MPC-MRP & 0.1010 & 0.68 & 7.23 & $1.1810^{4}$ & 36.69 & 4.51 & 32.71 \\
\hline
\end{tabular}

smaller $\bar{e}_{\text {MARTE }}\left(i_{d q}\right), u_{\mathrm{CMV}}, u_{z}, \bar{f}_{\mathrm{ASN}}$, calculation time $t_{c}$, and THD of current and output inverter voltage.

\section{Conclusion}

A robust model predictive control for multilevel inverter fed PMSM for electrical vehicle application is proposed in this paper. The computational delay is compensated using a proposed modified two-step horizon prediction. A proposed improved multiobjective cost function $f^{\text {dcom }}$ incorporates the control objectives and evaluates every sampling interval to generate the optimal switching state applied directly to the inverter without modulation stage. The time-varying behavior of the electrical vehicle system affects the good dynamic and steady-state performances of the standard finitecontrol-set model predictive control with improved cost function (FCS-MPC-ICF) control. Simulation results under different scenarios of parameters variation approve the effectiveness of the proposed robust FCS-MPC with "dynamic error correction" (DEC) and "modified revised prediction" (MRP) principles in terms of control robustness. The proposed robust FCS-MPC-DEC solution outperforms both the proposed robust FCS-MPC-MRP and standard FCS-MPCICF controls.

\section{Appendix}

\section{Bounded-Input Bounded-Output (BIBO) Stability Criteria}

With the assumption that the future "predicted" value will approach its "reference" $x_{p}(k+1)=x^{\text {ref }}(k+1)$ [27], the discrete transfer function between the measurement and the reference using equations (29) and (14) is obtained:

$$
\frac{x_{m}}{x^{\mathrm{ref}}}=\frac{\left(L_{s 0} / L_{s}\right) z+\left(1-\left(L_{s 0} / L_{s}\right)\right) \sigma_{1}}{\left(z-\left(1-\left(L_{s 0} / L_{s}\right)\right) \sigma_{1}\right):=F(z)} .
$$

Based on "bounded-input bounded-output (BIBO)" stability criteria, the system is stable, if and only if all roots of $F(z)=z-1+\sigma_{1}\left(L_{s 0} / L_{s}\right)$ are inside the unit circle, i.e.,

$$
|z|=\left|\left(1-\frac{L_{s 0}}{L_{s}}\right) \sigma_{1}\right|<1 \Longleftrightarrow 0<\frac{L_{s 0}}{L_{s}}<\underbrace{1+\frac{1}{\sigma_{1}}, \text { where, } 0<\sigma_{1} \leq 1}_{\chi_{\max }} .
$$

The stability range of inductance value $L_{s}$ in the controller is enlarged from the original/conventional solution $\left(0 ; 2 L_{s}\right)$ to $\left(0 ; \chi_{\max } L_{s}\right)$. 
In the original/conventional solution (i.e., the classical FCS-MPC solution), $\sigma_{1}=1$. So, the characteristic function becomes $F(z)=z-1+\sigma_{1}\left(L_{s 0} / L_{s}\right)$. Therefore, the BIBO stable requirement is $0<\left(L_{s 0} / L_{s}\right)<2$.

The stability of a sampled closed loop system, with the discrete-time format of $C(z) / R(z)=G(z) /(1-G H(z))$, can be determined by the location of its closed loop poles in $z$-plane, which are the roots of the characteristic equation $1+G H(z)=0$ as follows:

(i) System will be stable if the closed loop poles or the roots of the characteristic equation will lie within the unit circle of $|z|=1$ in the $z$-plane

(ii) System will be marginally stable if a pole or a pair of complex conjugate poles lie(s) on the circle of $|z|=1$

(iii) System will be unstable in other cases

\section{Data Availability}

No data were used to support this study.

\section{Conflicts of Interest}

The authors declare that there are no conflicts of interest regarding the publication of this paper.

\section{References}

[1] S. Zhang and X. Zhuan, "Model-predictive optimization for pure electric vehicle during a vehicle-following process," Mathematical Problems in Engineering, vol. 2019, Article ID 5219867, 15 pages, 2019.

[2] J. J. So, J. Kang, S. Park, I. Park, and J. Lee, "Automated emergency vehicle control strategy based on automated driving controls," Journal of Advanced Transportation, vol. 2020, Article ID 3867921, 11 pages, 2020.

[3] J. J. Justo, F. Mwasilu, E.-K. Kim, J. Kim, H. H. Choi, and J.-W. Jung, "Fuzzy model predictive direct torque control of ipmsms for electric vehicle applications," IEEE/ASME Transactions on Mechatronics, vol. 22, no. 4, pp. 1542-1553, 2017.

[4] K. Lu, Y. Zhu, Z. Wu, and M. Xiao, "Suppression of current fluctuations and the brake torque for pmsm shutoff in electric vehicles," Mathematical Problems in Engineering, vol. 2019, Article ID 5026316, 13 pages, 2019.

[5] A. Arias, E. Ibarra, E. Trancho, R. Griñó, I. Kortabarria, and J. Caum, "Comprehensive high speed automotive sm-pmsm torque control stability analysis including novel control approach," International Journal of Electrical Power \& Energy Systems, vol. 109, pp. 423-433, 2019.

[6] Z. Zhang, J. Rodriguez, and R. Kennel, "Robust predictive control of 3L-NPC converter fed pmsm drives for electrical car applications," in Proceedings of the 2018 IEEE Energy Conversion Congress and Exposition (ECCE), pp. 5204-5208, IEEE, Portland, OR, USA, September 2018.

[7] P. Thamizhazhagan and S. Sutha, "Adaptive vector control reference strategy based speed and torque control of permanent magnet synchronous motor," Microprocessors and Microsystems, vol. 74, Article ID 103007, 2020.

[8] X. Liu, C. Zhang, K. Li, and Q. Zhang, "Robust current control-based generalized predictive control with sliding mode disturbance compensation for pmsm drives," ISA Transactions, vol. 71, pp. 542-552, 2017.

[9] H. Laghridat, A. Essadki, and T. Nasser, "Comparative analysis between pi and linear-adrc control of a grid connected variable speed wind energy conversion system based on a squirrel cage induction generator," Mathematical Problems in Engineering, vol. 2019, Article ID 8527183, 16 pages, 2019.

[10] I. Aboudrar, S. El Hani, M. S. Heyine, and N. Naseri, "Dynamic modeling and robust control by adrc of grid-connected hybrid pv-wind energy conversion system," Mathematical Problems in Engineering, vol. 2019, Article ID 8362921, 19 pages, 2019.

[11] H. Li, S. Chen, X. Wu, and G. Tan, "Model predictive control method with constant switching frequency to reduce common-mode voltage for pmsm drives," Journal of Electrical and Computer Engineering, vol. 2018, Article ID 1090452, 12 pages, 2018.

[12] L. Sheng, D. Li, and Y. Ji, “Two-vector fcs-mpc for permanentmagnet synchronous motors based on duty ratio optimization," Mathematical Problems in Engineering, vol. 2018, Article ID 9061979, 9 pages, 2018.

[13] V.-Q.-B. Ngo, M.-K. Nguyen, T.-T. Tran, J.-H. Choi, and Y.-C. Lim, "A modified model predictive power control for grid-connected t-type inverter with reduced computational complexity," Electronics, vol. 8, no. 2, p. 217, 2019.

[14] B. Ding, X. Tang, and J. Hu, "A summary of dynamic output feedback robust mpc for linear polytopic uncertainty model with bounded disturbance," Mathematical Problems in Engineering, vol. 2020, Article ID 3830724, 19 pages, 2020.

[15] J.-F. Stumper, S. Kuehl, and R. Kennel, "Predictive torque control for ac drives: improvement of parametric robustness using two-degree-of-freedom control," in Proceedings of the 2013 IEEE Energy Conversion Congress and Exposition, pp. 1170-1175, IEEE, Denver, CO, USA, September 2013.

[16] T. Turker, U. Buyukkeles, and A. F. Bakan, "A robust predictive current controller for pmsm drives," IEEE Transactions on Industrial Electronics, vol. 63, no. 6, pp. 3906-3914, 2016.

[17] M. Siami, D. A. Khaburi, A. Abbaszadeh, and J. Rodriguez, "Robustness improvement of predictive current control using prediction error correction for permanent-magnet synchronous machines," IEEE Transactions on Industrial Electronics, vol. 63, no. 6, pp. 3458-3466, 2016.

[18] A. Sinha, K. Chandra Jana, and M. Kumar Das, "An inclusive review on different multi-level inverter topologies, their modulation and control strategies for a grid connected photovoltaic system," Solar Energy, vol. 170, pp. 633-657, 2018.

[19] A. Katkout, A. Essadki, and T. Nasser, "A modified multiobjective finite control set model predictive control for threelevel neutral-point clamped inverter," in Proceedings of the 2019 International Conference on Wireless Technologies, Embedded and Intelligent Systems (WITS), pp. 1-6, IEEE, Fez, Morocco, April 2019.

[20] A. Katkout, A. Essadki, and T. Nasser, "An improved multiobjective finite control set model predictive control for grid connected three-level neutral-point clamped inverter," in Proceedings of the 2019 International Conference on Advanced Communication Technologies and Networking (CommNet), pp. 1-6, Rabat, Morocco, April 2019.

[21] J. Rodriguez and P. Cortes, Predictive Control of PowerConverters and Electrical Drives, vol. 40, John Wiley \& Sons,Hoboken, NJ, USA, 2012. 
[22] J. Yan, H. Wang, S. Huang, and Y. Lan, "Disturbance observer-based backstepping control of PMSM for the mine traction electric locomotive," Mathematical Problems in Engineering, vol. 2018, Article ID 7253210, 10 pages, 2018.

[23] L. Wang, S. Chai, D. Yoo, L. Gan, and K. Ng, PID and Predictive Control of Electrical Drives and Power Converters Using MATLAB/Simulink, John Wiley \& Sons, Hoboken, NJ, USA, 2015.

[24] V. Q. B. Ngo, Algorithmes de conception de lois de commande prédictives pour les systèmes de production d'énergie, Ph.D. thesis, University of Paris-Saclay, France, 2017.

[25] A. Choudhury, P. Pillay, and S. S. Williamson, "Modified dcbus voltage balancing algorithm for a three-level neutralpoint-clamped pmsm inverter drive with reduced commonmode voltage," IEEE Transactions on Industry Applications, vol. 52, no. 1, pp. 278-292, 2016.

[26] V.-H. Vu, V.-T. Pham, H.-N. Nguyen et al., "Lyapunov-induced model predictive power control for grid-tie three-level neutral-point-clamped inverter with dead-time compensation," IEEE Access, vol. 7, pp. 166869-166882, 2019.

[27] A. Mbarek and K. Bouzrara, "Fault tolerant control for mimo nonlinear systems via mpc based on mimo arx-laguerre multiple models," Mathematical Problems in Engineering, vol. 2019, Article ID 9012182, 26 pages, 2019. 